SANHUEZA, Guillermo; PÉREZ, Francisca "Cárceles concesionadas en Chile: evidencia empírica y perspectivas futuras a 10 años de su creación".

Polít. crim. Vol. 12, No 24 (Diciembre 2017), Art. 11, pp. 1066-1084.

[http://www.politicacriminal.cl/Vol_12/n_24/Vol12N24A11.pdf]

\title{
Cárceles concesionadas en Chile: evidencia empírica y perspectivas futuras a 10 años de su creación
}

\section{Prison concessions in Chile: empirical evidence and future prospects 10 years after their creation}

\author{
Guillermo Enrique Sanhueza \\ $\mathrm{PhD}$ Trabajo Social y Sociología, U. of Michigan \\ Investigador Facultad de Ciencias Sociales y Humanidades \\ Universidad San Sebastián \\ guillermo.sanhueza@uss.cl \\ Francisca Pérez \\ Socióloga Pontificia Universidad Católica de Chile \\ Investigadora asociada al Centro de Políticas Públicas UC \\ fperez5@uc.cl
}

\section{Resumen}

El presente artículo analiza el sistema de concesiones penitenciarias en Chile a 10 años de su creación con el fin de contribuir a la discusión pública respecto al futuro de este ámbito de la política penitenciaria. Para ello, se utilizan datos secundarios de diversas fuentes, incluyendo el Estudio de Reincidencia de Paz Ciudadana y la Primera Encuesta de Calidad de Vida Penitenciaria. Nuestros hallazgos sugieren que (i) existe poca evidencia empírica respecto al funcionamiento de las cárceles concesionadas, (ii) estos recintos ofrecerían mejores condiciones de infraestructura y habitabilidad en algunos indicadores, (iii) las cárceles concesionadas exhibirían indicadores más deficitarios que sus contrapartes públicas en cuanto a acceso a servicios de salud, relaciones internos/ funcionarios, maltrato a internos y acceso a programas de reinserción. A pesar del carácter no-concluyente de la evidencia presentada en este estudio, nuestro análisis sugiere que existen diversos aspectos del funcionamiento y desempeño de las cárceles concesionadas que deberían revisarse pues, este sistema llevaba la promesa de ofrecer "mejor reinserción social" que el sistema tradicional y a una década parece ser que esta promesa sigue sin cumplirse. Finalmente, se proponen algunos puntos para alimentar la discusión en torno al rol de las cárceles concesionadas en la actualidad.

Palabras clave: cárceles, Chile, sector privado, reinserción social, desempeño.

\begin{abstract}
This article analyzes the system of prison concessions in Chile 10 years after its creation in order to contribute to the public discussion regarding the future of this area of prison policies. To this end, secondary data are used from a variety of sources, including the
\end{abstract}


SANHUEZA, Guillermo; PÉREZ, Francisca "Cárceles concesionadas en Chile: evidencia empírica y perspectivas futuras a 10 años de su creación”.

Recidivism Study (Paz Ciudadana) and the First Survey of Prison Life. Our findings suggest that (i) there is little empirical evidence regarding the operation of private prisons, (ii) these facilities would offer better infrastructure and habitability conditions for some indicators, (iii) privately-operated prisons would exhibit more deficient indicators than their public counterparts in access to health services, internal relations / officials, mistreatment of inmates and access to rehabilitative programs. Despite of the non-conclusive evidence presented in this study, our analysis suggests that there are several aspects of the functioning and performance of the franchised establishments that should be reviewed, since this system promised to offer "better social reintegration" than the traditional, public one. After a decade it seems that this promise remains unfulfilled. Finally, some points are proposed to feed the discussion about the role of jails currently under concession.

Key words: prisons, Chile, private sector, rehabilitation, performance.

\section{Introducción}

El sistema de concesiones carcelarias acaba de cumplir 10 años de su instalación en Chile. La promesa inicial del sistema de concesiones era la creación de una alianza estratégica entre el sector público y privado para generar sistemas carcelarios flexibles, creativos, rentables, eficientes, de mayor calidad y de menor costo respecto a sus contrapartes públicas. Lo que se buscaba lograr, era renovar los establecimientos y ciertas dinámicas de ellos para lograr estándares de calidad más altos, enfrentar los problemas de sobrepoblación, optimizar la seguridad en los recintos y mejorar los programas de reinserción ${ }^{1 \_2}{ }^{3}$.

El sector privado se constituiría, así, como el aliado estratégico del quehacer estatal en temas de política penitenciaria, posibilitando que los objetivos de rehabilitación sean cumplidos de manera más eficiente. Pues como sostiene Pavić, el debate e implementación de cárceles privadas solamente surge en contextos en los que el modelo tradicional no cumple con las expectativas de funcionamiento ${ }^{4}$.

Algunos han abogado, precisamente, por una expansión del mismo como respuesta a los problemas de hacinamiento y gestión que tendrían las cárceles públicas, confiando en la capacidad de los actores privados para solucionar las condiciones de habitabilidad de las prisiones públicas, sus problemas de hacinamiento y de gestión, mejorar la eficiencia y capacidad organizacional, lograr una mayor flexibilidad y apostar por el aporte que la

\footnotetext{
${ }^{1}$ ARRIAGADA, Isabel. "De cárceles y concesiones: Privatización carcelaria y penalidad neoliberal.", Revista de Derecho (Valdivia) Vol. XXV, N² (2012), p. 28.

2 PRADO, Francisco, "El sistema penitenciario chileno, una aproximación crítica”, Revista Electrónica Agenda Pública Vol. V (2006), pp. 2-9.

3 MARTÍNEZ, Fernando; ESPINOZA, Olga, "Cárceles concesionadas en Chile: ¿El camino a la privatización?”, Revista electrónica Debates Penitenciarios, Área de Estudios Penitenciarios CESC, Vol. 9 (2009), pp. 1-18.

${ }^{4}$ PAVIĆ, Ivica, "Perspectives of Prison Privatization as a Solution to the Prison System Crisis in Croatia", European Journal of multidisciplinary studies $\mathrm{Vol} 3, \mathrm{~N}^{\circ} 1$ (2016).
} 
experiencia y capacidad innovadora del sector privado, los que contribuyen al quehacer de Gendarmería ${ }^{5}{ }^{6}$.

Otros han asociado el rol de los privados en las cárceles chilenas a una "hipertrofia del estado penal", impulsando de manera excesiva el rol del estado en materia de manejo carcelario producto del incumplimiento de las expectativas que se preveían del sistema concesionado en términos de reinserción y calidad de vida.

Sin embargo, pocos estudios empíricos en nuestro país han abordado el funcionamiento del sistema de concesiones carcelarias. Así, el propósito de este artículo será revisar la evidencia desde datos secundarios para contribuir a este debate, promoviendo así un análisis de la situación actual de las cárceles concesionadas a 10 años de su implementación y en referencia a las expectativas que se tenían de este sistema.

Este artículo comienza con una revisión de antecedentes y una descripción del modelo de concesiones carcelarias en términos generales y sus particularidades en Chile; luego, analiza diversos "indicadores de desempeño" (infraestructura, acceso a programas, niveles de maltrato, reincidencia y otros) provenientes de la evidencia desde distintas fuentes secundarias, incluyendo el Estudio de Reincidencia de Fundación Paz Ciudadana ${ }^{7}$ y la Primera Encuesta de Percepción de Calidad de Vida Penitenciaria ${ }^{8}{ }^{9}$. Luego, en base a los datos disponibles, se analizan algunas fortalezas, nudos críticos y desafíos del sistema de concesiones. Finalmente, se elaboran algunas conclusiones de este estudio y se realizan algunas recomendaciones de política pública.

\section{Discusión bibliográfica}

\subsection{El rol de los privados en las cárceles}

Las funciones de las cárceles privadas en el mundo dicen relación con generar alternativas adicionales al modelo carcelario clásico, en cual son instituciones estatales quienes se hacen cargo de manera exclusiva de los centros penitenciarios, muchas veces resolviendo el problema de la seguridad en las sociedades desde diferentes grados de eficacia y buscando que los actores sociales cooperen en conjunto. Así, se le quita al estado el deber exclusivo y

\footnotetext{
${ }^{5}$ PRATT, Travis; MAAHS, Jeff, “Are Private Prisons More Cost-Effective Than Public Prisons? A MetaAnalysis of Evaluation Research Studies”, Crime \& Delinquency Vol. 45, N 3 (1999), pp. 358-371.

6 ARELLANO, Jaime, "Reforma Penitenciaria: El caso del programa de concesiones en infraestructura penitenciaria en Chile" en : Seminario Interamericano "Justicia y Gobernabilidad Democrática" (2003) Santiago, Chile, p. 6.

7 FUNDACION PAZ CIUDADANA Y BID, "Evaluación del sistema concesionado versus el sistema tradicional en la reducción de la reincidencia delictual", 2013, disponible en: http://www.pazciudadana.cl/wp-content/uploads/2013/07/2013-07-02_Evaluaci\%C3\%83\%C2\%B3n-delsistema-concesionado-versus-el-sistema-tradicional-en-la-reducci\%C3\%83\%C2\%B3n-de-la-reincidenciadelictual.pdf [última visita Agosto 2017].

${ }^{8}$ SANHUEZA, Guillermo, "Reinserción Social: el desempeño moral de las cárceles en Chile", Nova Criminis $\mathrm{N}^{\circ} 9$ (2015), pp. 181-209.

9 ESPINOZA, Olga; MARTÍNEZ, Fernando; SANHUEZA, Guillermo, "El sistema penitenciario y su impacto en las personas privadas de libertad", en: VV.AA., Informe sobre los Derechos Humanos en Chile (2014).
} 
SANHUEZA, Guillermo; PÉREZ, Francisca "Cárceles concesionadas en Chile: evidencia empírica y perspectivas futuras a 10 años de su creación”.

el monopolio de la administración carcelaria ${ }^{10}$. Sin embargo, existen diversos factores y contextos que inciden al momento de escoger la estrategia concesionada como opción de política carcelaria.

En el mundo existen fundamentalmente tres diferentes modalidades de participación de los actores privados en instituciones penitenciarias: i) en primer lugar, está el sistema de leasing, muy utilizado en países latinoamericanos y en el cual la alianza con privados se da de manera que éstos diseñan, construyen y equipan los establecimientos penitenciarios sin intervenir en la operación del recinto, ii) la segunda modalidad es la de privatización, en la cual se delega en forma absoluta la administración del penal a los actores privados, relegando el rol estatal a la mínima intervención reguladora; este modelo se observa en Estados Unidos, e Inglaterra, iii) una tercera modalidad es de carácter mixto y consiste en la delegación a privados una parte importante de las tareas del penal o una "terciarización" de ciertas tareas, dejando a cargo de Gendarmería (o su equivalente en otro país) la facultad exclusiva de vigilancia y castigo.

Esta última modalidad "mixta" es la utilizada en algunas cárceles francesas y es la que fue adaptada para ser aplicada en nuestro país, generando de esa manera una alianza públicoprivada en la cual las responsabilidades quedan reguladas a partir de una Base de Licitación y un posterior contrato de licitación por 20 años. Así, en Chile, el sistema de cárceles privadas funciona en base a una concesión otorgada a un privado para que dirija y administre una unidad penal, aunque esta sigue bajo la custodia de Gendarmería de Chile y, de manera más general, bajo la responsabilidad del estado. Se establece, así, una relación contractual en términos amplios, abriendo posibilidades a que existan múltiples formas de llevarse a cabo bajo diferentes contratos, estándares o características.

La experiencia internacional respecto a la privatización de las cárceles nos muestra que en Gran Bretaña, algunas cárceles tienen un régimen privado que abarca la mayoría de sus aspectos: intervienen en la construcción, manejo de las cárceles y contratación de personal. En este país, la privatización carcelaria surgió en un periodo en que se aplicaron múltiples medidas neoliberales producto de una amplia fe en la competencia, las empresas y los incentivos; pero también con una amplia fe en este sistema desde el debate académico y político. Así, una de las medidas de Margaret Thatcher se basó en una consultoría que consideró que la mejor opción era la intervención privada no solo en la construcción de las cárceles, sino también en su manejo y la contratación de personal. Actualmente, son 11 las cárceles manejadas por privados en ese país.

En el caso de España, no resulta riguroso hablar de privatización, a pesar de que la participación estatal va disminuyendo cada vez más. Los orígenes de la participación de privados en el sector penitenciario se remonta a los siglos XIX y XX. Sin embargo, en los ochenta ha comenzado una progresiva colaboración privada en los recintos penitenciarios, lo cual perdura hasta hoy en ámbitos de construcción, contabilidad, cocina y cafetería como servicios proveídos por empresas privadas.

\footnotetext{
${ }^{10}$ BURÓN, Javier Nistal, "El futuro de la cárcel y la cárcel del futuro", Quadernos de criminología: revista
} de criminología y ciencias forenses $\mathrm{N}^{\circ} 20$ (2013), pp. 34-41. 
Polít. crim. Vol. 12, Nº 24 (Diciembre 2017), Art. 11, pp. 1066-1084.

[http://www.politicacriminal.cl/Vol_12/n_24/Vol12N24A11.pdf]

En Francia coexiste un formato privado con uno público: el gobierno mantiene y contrata a los internos mientras las empresas diseñan, construyen y equipan la infraestructura; además del subcontrato de algunos servicios bajo la idea de que esta división de tareas permite que cada uno de los actores se enfoque de manera total a su quehacer. Así, coexisten a la vez cárceles administradas y gestionadas de manera centralizada, como también un sistema mixto en el cual el gobierno se encarga del mantenimiento y control de los internos y los privados diseñan, construyen y equipan las instalaciones penitenciarias, subcontratando los otros servicios adicionales exceptuando la seguridad. Este sistema funciona desde los 90's y hacia el año 2007 los establecimientos de tipo mixto albergaban al $25 \%$ del total de la población penitenciaria.

\subsection{Voces a favor $y$ en contra}

Algunas voces a favor de las alianzas público-privadas de los centros penitenciarios argumentan que la concesión del sistema penal permite reunir estrategias del sector privado y del público, construyendo de esa manera una gestión mixta en que los privados se encarguen del aspecto económico-administrativo y la ejecución penal sea de carácter público. A su vez, en un análisis más económico desde la Public Choice Theory, existe la expectativa de que los privados sean más eficientes y racionales en la prestación de servicios carcelarios que los organismos públicos: pues la competencia impulsaría una mayor eficiencia administrativa y también permite evitar que los internos estén forzados a aceptar una peor calidad de servicios por provenir desde un único prestador público.

Otras voces promotoras han señalado que un manejo privado de las cárceles disminuiría los aspectos burocráticos y traería como consecuencia una mayor flexibilidad respecto a la satisfacción de necesidades ${ }^{11}$. Además de los argumentos relativos a la eficiencia y al costoefectividad de este tipo de alianzas, la flexibilidad y dinamismo resulta un argumento a favor de la participación de los privados: el sector privado respondería de manera más rápida y adecuada las necesidades, promoviendo la creatividad, corrigiendo los problemas y dando más espacio a nuevas ideas sin una estructura tan burocrática.

A su vez, hay quienes destacan que esta alianza fortalece la rendición de cuentas y transparencia respecto a las instancias únicamente estatales, además de disminuir los espacios para corrupciones. Finalmente, un último argumento a favor de las alianzas público-privadas es que los sistemas de seguridad implementados y gestionados por los privados contarían con profesionalismo, tecnología y entrenamiento de mayor efectividad que los servicios públicos.

Entre quienes critican el sistema privado, han señalado que no existiría una disminución efectiva de los costos a largo plazo, pues los privados deberían incurrir en gastos operativos y de gestión que el sistema público tiene cubiertos de antemano; en este sentido, debido a que el estado tradicionalmente se ha hecho cargo de las cárceles, suele tener una experticia acumulada en el tema que incluye una estimación y consideración de gastos operativos

${ }^{11}$ DUNCAN, Jeanine, "Report on corrections and the private sector", en: Comission on criminal and juvenile justice, 1986, disponible en: https://www.ncjrs.gov/pdffiles1/Photocopy/102380NCJRS.pdf [última visita Agosto 2017]. 
SANHUEZA, Guillermo; PÉREZ, Francisca "Cárceles concesionadas en Chile: evidencia empírica y perspectivas futuras a 10 años de su creación”.

tales como salarios, capacitaciones, sistemas operativos y de gestión; los privados, por otra parte, carecerían de esa experticia y tendrían que entrar a conocer e invertir en una variedad de ítems que no son inmediatos de estimar.

En términos de su desempeño moral, un estudio comparativo llevado a cabo por Liebling y Crewe el año 2012 revela que las cárceles de carácter público obtuvieron mejores resultados que su comparación entre los privados, principalmente en lo relativo a la lealtad y profesionalismo del personal. Así también, las cárceles privadas tendrían comparativamente un mejor trato y mayores niveles de respeto que en las cárceles tradicionales. Por otro lado, ellos encontraron menores niveles de profesionalismo en los equipos técnicos de las cárceles privadas; menor profesionalismo del staff; una alta y mayor rotación del personal; una débil oferta de reinserción; mayor percepción de injusticias por parte del staff; y también mayores debilidades en materias de seguridad y control, lo cual según los autores vuelve a las prisiones privadas peores que sus contrapartes estatales a nivel agregado.

Además, según los autores, en estos establecimientos se replicarían problemas de la cultura carcelaria y de drogas presentes también en las cárceles tradicionales ${ }^{12}$. Sin embargo, la cultura organizacional resulta muy diferente entre ambos sistemas y desde la evidencia de Liebling y Ludlow, es más estable y positiva en el caso de las cárceles públicas, con menores índices de estrés y frustración. Además, un mayor uso de autoritarismo estaría presente entre los privados que en los organismos públicos ${ }^{13}$.

\subsection{Antecedentes y descripción del modelo de concesiones carcelarias en Chile}

En Chile, el sistema de concesión de cárceles nace en el año 2000 como respuesta a los graves problemas de hacinamiento que enfrentaba el sistema penitenciario en aquel entonces. En noviembre del año 2005 se inaugura el primer centro penitenciario concesionado en la ciudad de Rancagua, dando así cumplimiento al primer paso de uno de los ejes principales de la política pública en la agenda de justicia 2006-2010 y viéndose secundado por la concreción de los demás centros concesionados que existen actualmente. La idea era contar con una mejor infraestructura, que permita una segregación penal para evitar el "contagio criminógeno", el cual sería inherente a todo sistema penitenciario mal operado.

Además, se esperaba de estas instituciones una mejor capacidad para adaptarse a realidades locales, una mayor inversión en tecnología y seguridad, contar con ventajas comparativas de gestión, lograr una mayor eficiencia y mejorar la calidad de vida y el hacinamiento que caracterizan a las cárceles tradicionales chilenas ${ }^{14}$.

\footnotetext{
${ }^{12}$ LIEBLING, Alison; CREWE, Ben, "Prisons beyond the New Penology: The Shifting Moral Foundations of Prison Management" en: SIMON, Jonathan; SPARKS, Richard (Eds.), The Sage Handbook of Punishment and Society, Londres: Sage Publications Ltd., 2012, pp. 283-307

${ }^{13}$ LIEBLING, Alison; LUDLOW, Amy, "Privatising public prisons: Penality, law and practice", Australian \& New Zealand Journal of Criminology (2016).

${ }^{14}$ RIBERA, Teodoro, "Realidad actual y desafíos del sistema penitenciario chileno", en: VV.AA., Seminario Cárceles concesionadas revitalizando el modelo organizado por el Instituto Libertad y Desarrollo (2012). Santiago, Chile.
} 
Polít. crim. Vol. 12, Nº 24 (Diciembre 2017), Art. 11, pp. 1066-1084.

[http://www.politicacriminal.cl/Vol_12/n_24/Vol12N24A11.pdf]

El modelo chileno de concesiones penitenciarias correspondería a un modelo mixto donde mientras la custodia corre por cuenta de Gendarmería de Chile, la intervención psicosocial y la provisión de servicios a la población penal están a cargo de la empresa concesionaria. Este surgió de la promesa de avanzar en eficiencia y enfocar los esfuerzos de rehabilitación a la población penal, resguardando la seguridad ciudadana y los derechos fundamentales de los internos, modernizando las dependencias penales y la segmentación de los internos y planteándose la misión de generar proyectos de capacitación y reinserción social más efectivos.

Así, los contratos entre los actores privados y las instituciones públicas serían del tipo B.O.T (Build, Operate and Transfer), que consiste principalmente en que un privado construya y explote la obra pública y, finalizada la concesión, le entregue la obra al Estado para que lo concesione nuevamente. Este modelo se implementó en el marco de una búsqueda por aumentar la capacidad de innovación de las cárceles, para rescatar los aportes en eficiencia del sector privado y poder desempeñar de mejor manera aquellos ámbitos que queden relegados al estado. Se buscaba también aumentar los actores participantes en materia de centros penitenciarios. Finalmente también se buscaba focalizar y/u optimizar los recursos materiales, económicos y humanos de los que dispone el estado.

De esta forma, la división de roles quedó concretada en que el Estado se encarga de la vigilancia, seguridad y administración del establecimiento (a cargo de Gendarmería de Chile), y debe fiscalizar y controlar el cumplimiento de estándares, además de pagar semestralmente a los concesionarios. Por su parte, los actores privados deben diseñar, construir y equipar los establecimientos, generar un manual operativo para capacitar al personal de Gendarmería, mantener la infraestructura y equipamiento, proveer servicios alimenticios, de salud y de reinserción social.

Actualmente, existen tres grupos de concesiones privadas operando cárceles en nuestro país. El grupo 1 está compuesto por los centros penitenciarios de Alto Hospicio, La Serena y Rancagua, los cuales funcionan desde el año 2006 y tienen capacidad para más de 5.000 internos. El grupo 2 opera los penales de Antofagasta y Concepción, con capacidad para 2.350 internos. Finalmente el grupo 3 desarrolla su trabajo en los penales de Santiago 1, Valdivia y Puerto Montt, los cuales operan desde el año 2007 con capacidad para más de 5.000 internos.

En términos de la población que albergan, las cárceles concesionadas en el país (al 31 de Enero del 2016) concentraban a un 24,6\% del total de condenados del país y a un 48,2\% del total de imputados/as, con 7.617 internos condenados y poco más de 6.200 imputados ${ }^{15}$.

En referencia a la implementación de este sistema en Chile, Arriagada ${ }^{16}$ sostiene que la estrategia de cárceles concesionadas se origina como parte de una serie de políticas

\footnotetext{
${ }^{15}$ Gendarmería de Chile. "Boletín Estadístico Chile: Situación carcelaria en Cifras", Nº1, octubre de 2016, disponible en http://static.emol.cl/emol50/documentos/archivos/2016/11/02/20161102115114.pdf [última visita 30 noviembre 2016].

${ }^{16}$ ARRIAGADA, Isabel. "De cárceles y concesiones: Privatización carcelaria y penalidad neoliberal.", Revista de Derecho (Valdivia) Vol. XXV, N 2 (2012).
} 
SANHUEZA, Guillermo; PÉREZ, Francisca "Cárceles concesionadas en Chile: evidencia empírica y perspectivas futuras a 10 años de su creación”.

neoliberales y un espíritu basado en nociones de que es el sector privado el encargado de complementar el quehacer del estado. Así, la finalidad de la privatización carcelaria era sustituir los defectos administrativos del estado y entregar soluciones más eficaces, generando un espacio para la contribución de los privados en el manejo de los centros penitenciarios. Esto a su vez pone sobre la mesa un debate ético respecto a la existencia de lucro en el castigo, así como un cuestionamiento a la manera en que el sector público se está haciendo cargo del tema.

El rol del sector privado en las cárceles y los debates asociados a este han sido largamente discutidos en mayor profundidad en otros trabajos previos, tanto del extranjero como de Chile $^{17}$. Sin embargo, existen pocos trabajos que presenten evidencia empírica respecto a algunos aspectos de funcionamiento de las cárceles concesionadas en Chile. Nuestro trabajo ofrece una revisión de fuentes secundarias que analizan el rol de los privados en las cárceles.

\section{Metodología}

Este artículo hace un análisis de fuentes y datos secundarios para analizar empíricamente el sistema de concesiones en Chile y su funcionamiento en base a diversos indicadores proxies de desempeño, como son las condiciones de habitabilidad, el acceso a programas, los niveles de reincidencia, la violencia entre internos al interior de los penales, y los niveles de maltrato de funcionarios del estado hacia los reclusos. Las fuentes principales de análisis fueron la Primera Encuesta de Percepción de Calidad de Vida Penitenciaria, el estudio de reincidencia de Paz Ciudadana y el BID, el estudio de reincidencia de Gendarmería $^{18}$, el análisis de Oliveri y la investigación de Martínez y Espinoza, entre otros.

La Primera Encuesta de Percepción de Calidad de Vida Penitenciaria fue llevada a cabo el año 2013 a 2.093 personas privadas de libertad de 75 cárceles chilenas a lo largo del país. De esos 75 centros penitenciarios, 69 se regían por el sistema tradicional y 6 eran centros concesionados (Alto Hospicio, La Serena, Rancagua, Biobío, Valdivia, Puerto Montt). En términos totales, hubo un $77,7 \%$ de tasa de respuesta, logrando un $71,2 \%$ de tasa de respuesta en cárceles concesionadas $(\mathrm{n}=228)$.

\section{Resultados: análisis descriptivo}

La Tabla 1 muestra los principales estadísticos descriptivos de indicadores de Habitabilidad (percepción sobre la infraestructura y habitabilidad; acceso y calidad de los servicios de salud; y el acceso y trato a las visitas), Violencia Carcelaria (niveles de maltrato físico y psicológico experimentados al interior de la cárcel; percepción sobre la

17 OLIVERI, Katherine, "Programas de rehabilitación y reinserción de los sistemas de cárceles", Sociedad Chilena de Políticas Públicas (2011) Santiago, Chile, disponible en: http://www.sociedadpoliticaspublicas.cl/archivos/BLOQUET/Seguridad_Ciudadana_y_Justicia/Programas\%2 0de\%20rehabilitacion\%20y\%20reincersion\%20en\%20carceles\%20concesionadas.pdf [última visita Agosto2017].

${ }^{18}$ MORALES, Ana María, et al., "La Reincidencia en el Sistema Penitenciario Chileno”, Santiago: Fundación Paz Ciudadana, 2013. 
calidad de la relación con los funcionarios uniformados; violencia entre internos; violencia desde funcionarios; y presencia de castigos y violencia en los allanamientos) y el Acceso a programas de reinserción en la cárcel, comparándolos según se trate de un sistema tradicional o uno concesionado mediante una prueba $\mathrm{T}$ de Student para muestras independientes.

Tabla 1: Estadísticos descriptivos de habitabilidad, violencia y acceso a programas, según tipo de cárcel

\begin{tabular}{|c|c|c|c|c|c|c|c|}
\hline \multirow[t]{2}{*}{ Variable } & \multicolumn{3}{|c|}{ Cárceles Concesionadas } & \multicolumn{3}{|c|}{ Cárceles Tradicionales } & \multirow{2}{*}{$\begin{array}{c}\begin{array}{r}\text { Prueba t } \\
\text { diferencia } \\
\text { de medias }\end{array} \\
\begin{array}{c}\text { Sig. } \\
(\text { Valor } p)\end{array} \\
\end{array}$} \\
\hline & Media & $\begin{array}{c}\text { Desv. } \\
\text { Estándar }\end{array}$ & $\mathrm{N}$ válido & Media & $\begin{array}{l}\text { Desv. } \\
\text { Est. }\end{array}$ & $\mathrm{N}$ válido & \\
\hline Infraest./ habitabilidad & 5,3 & 2,1 & 203 & 5,4 & 2,5 & 1.668 & 0,6 \\
\hline Servicios de salud & 2,4 & 0,7 & 220 & 2,2 & 0,8 & 1810 & $0,0 * * *$ \\
\hline Acceso y trato a visitas & 1,9 & 0,8 & 213 & 2,3 & 0,8 & 1.755 & $0,0 * * *$ \\
\hline Niveles de maltrato & 1,5 & 1,4 & 217 & 1,3 & 1,3 & 1.771 & $0,0 * *$ \\
\hline Relación con funcionarios & 2,0 & 0,8 & 207 & 1,8 & 0,8 & 1.715 & $0,0 * * *$ \\
\hline Violencia entre internos & 0,4 & 0,5 & 219 & 0,4 & 0,5 & 1.796 & 0,9 \\
\hline funcionario- & 0,6 & 0,5 & 219 & 0,5 & 0,5 & 1787 & $0,0 * *$ \\
\hline Presencia de castigos & 0,5 & 0,4 & 219 & 0,4 & 0,5 & 1825 & $0,0 * *$ \\
\hline Violencia en allanamientos & 0,5 & 0,6 & 211 & 0,5 & 0,5 & 1671 & 0,2 \\
\hline Acceso a programas & 1,5 & 1,6 & 217 & 1,8 & 1,7 & 1.728 & $0,0 * *$ \\
\hline
\end{tabular}

Fuente: Elaboración propia a partir de la Primera Encuesta de Percepción de Calidad de Vida Penitenciaria 2013. Las cárceles concesionadas de la muestra = Alto Hospicio, La Serena, Rancagua, Bio-Bio, Valdivia y Puerto Montt (**) valor significativo $95 \%$ de confianza - (***) valor significativo $99 \%$ de confianza.

\subsection{Habitabilidad}

Los estadísticos descriptivos de las variables elegidas para este estudio muestran que, en las cárceles concesionadas, existe una percepción sobre la infraestructura penitenciaria cuyo promedio es de 5,3, la cual es bastante cercana al valor promedio del sistema en general (5,4 en una escala 0-9 donde valores más altos indican una mejor percepción) y al promedio de 5,4 presente entre las cárceles tradicionales. Estas diferencias no resultan estadísticamente significativas al 95\% de confianza $(\mathrm{p}=0,5 ; \mathrm{t}=0,5)$.

A pesar de que no se reportaron diferencias estadísticamente significativas en el indicador agregado de la percepción de infraestructura y habitabilidad, al examinar el detalle y de manera desagregada dicho índice, se observan diferencias entre ambos sistemas para las variables temperatura, calidad de comida y cantidad de la comida, aspectos en los cuales las cárceles tradicionales se posicionan mejor en comparación a las concesionadas. (Ver tabla 2). Sin embargo, en lo relativo a la presencia de baños en buen estado, limpieza del lugar en que duerme y acceso diario a una ducha, desde la evidencia de la encuesta de Percepción de calidad de vida penitenciaria es posible constatar que las cárceles concesionadas presentan una ventaja significativa en relación a las tradicionales (Ver tabla 2). 
SANHUEZA, Guillermo; PÉREZ, Francisca "Cárceles concesionadas en Chile: evidencia empírica y perspectivas futuras a 10 años de su creación”.

Tabla 2: Indicadores desagregados de percepción de infraestructura y habitabilidad según tipo de cárcel

\begin{tabular}{lccccc}
\hline \multicolumn{1}{c}{ Ítem } & $\%$ Total & $\begin{array}{c}\text { \% Cárcel } \\
\text { Concesionada }\end{array}$ & $\begin{array}{c}\text { \% Cárcel } \\
\text { Tradicional }\end{array}$ & Sig & N total \\
Temperatura & $33,2 \%$ & $20,6 \%$ & $34,7 \%$ & $* * *$ & 2.035 \\
Espacio suficiente en la celda & $41,9 \%$ & $46,7 \%$ & $41,4 \%$ & 2.029 \\
Baño en buen estado & $48,5 \%$ & $69,6 \%$ & $45,9 \%$ & $* * *$ & 2.049 \\
Ventilación adecuada & $51,0 \%$ & $54,2 \%$ & $50,6 \%$ & 2.043 \\
Lugar limpio para dormir & $60,3 \%$ & $82,0 \%$ & $57,7 \%$ & $* * *$ & 2.041 \\
Calidad de la comida & $66,4 \%$ & $46,8 \%$ & $68,8 \%$ & $* * *$ & 2.035 \\
Luz suficiente & $72,3 \%$ & $76,8 \%$ & $71,8 \%$ & & 2.055 \\
Cantidad de comida & $73,4 \%$ & $41,7 \%$ & $77,3 \%$ & $* * *$ & 2.044 \\
Acceso a ducha diaria & $89,7 \%$ & $95,9 \%$ & $88,9 \%$ & $* * *$ & 2.012 \\
\hline
\end{tabular}

Fuente: Elaboración propia a partir de Encuesta de Percepción de Calidad de Vida en Personas Privadas de Libertad 2013. (*) Significancia al 90\% de confianza (**) Significativo al $95 \%$ de confianza $(* * *)$ Significativo al $99 \%$ de confianza.

En lo referente al acceso y calidad de los servicios de salud que reciben los internos, el valor promedio para las cárceles concesionadas fue de 2,4 , lo cual se contrapone a un promedio nacional que alcanzó 2,3 (en una escala de 1 a 3 donde valores más altos indican una percepción de peores servicios de salud). Las cárceles tradicionales consideradas en la muestra tuvieron un promedio de 2,2 en el indicador de salud, lo que evidencia que existen diferencias estadísticamente significativas $(\mathrm{p}=0,0 ; \mathrm{t}=-2,9)$ que muestran peores percepciones del servicio de salud en las cárceles concesionadas que en las tradicionales.

Por otro lado, en el caso de acceso y trato a las visitas (Variable cuya escala de 1 a 3 donde los valores más altos representan mejor acceso y trato a visitas), el promedio de las cárceles concesionadas es de 1,9 y en las cárceles tradicionales es de 2,3. Esto quiere decir que en lo respectivo a las visitas, las cárceles concesionadas reportan significativamente peores resultados que las tradicionales de la muestra (al 95\% de confianza, donde $p=0,0 ; t=6,0$ ).

\subsection{Violencia carcelaria}

Asimismo, en cuanto a los niveles de maltrato físico y psicológico experimentado al interior de la cárcel el valor promedio para las cárceles concesionadas fue de 1,5 versus un promedio nacional que alcanzó 1,4 (en una escala de 0-4 donde valores más altos indican una percepción de mayor maltrato). Las cárceles tradicionales consideradas en la muestra tuvieron un promedio de 1,3 en el indicador de maltrato, lo que deja en evidencia que este tipo de malos tratos se darían con mayor fuerza en el caso de las cárceles concesionadas que en su contraparte estatal, de manera significativa al 95\% de confianza $(p=0,0 ; t=-2,0)$.

En cuanto a la media de percepción sobre la calidad de la relación con los funcionarios uniformados, los datos muestran un valor de 2,0 para las cárceles privadas (en una escala 13 , donde 1 es "buena relación" y 3 constituye "mala relación"), lo cual es superior al 1,8 de promedio nacional en la muestra. En el caso de las cárceles tradicionales, el promedio es de 1,8 , lo que mediante un test $\mathrm{t}$ al $95 \%$ de confianza resulta significativamente menor al de las 
Polít. crim. Vol. 12, Nº 24 (Diciembre 2017), Art. 11, pp. 1066-1084.

[http://www.politicacriminal.cl/Vol_12/n_24/Vol12N24A11.pdf]

cárceles concesionadas $(\mathrm{p}=0,0 ; \mathrm{t}=-3,5)$, dejando en evidencia que la relación con funcionarios sería peor en los centros concesionados que en el sistema tradicional.

Por otro lado, los auto-reportes de internos/as en cuanto a haber sufrido ya sea violencia física o psicológica de parte de otros internos(as) no presentó diferencias estadísticamente significativas entre ambos sistemas. Esto es un punto a favor de las cárceles concesionadas y que podría explicarse por las mejores condiciones de infraestructura y segregación. Lo mismo ocurre con la presencia de violencia durante los allanamientos, lo que no muestra diferencias estadísticamente significativas.

Respecto al maltrato de parte de funcionarios, se reportan promedios más bajos en las cárceles tradicionales que en las concesionadas de la muestra, lo cual considerando la escala de la variable ( 0 indica ausencia de malos tratos desde funcionarios y 1 indica la presencia de estos), posiciona a las concesionadas (promedio 0,6) en un panorama de mayor violencia desde los funcionarios que las cárceles tradicionales (promedio 0,5), significativo al $95 \%$ de confianza $(\mathrm{p}=0,0 ; \mathrm{t}=-2,2)$.

La presencia de castigos ( 0 indica ausencia de estos y 1 presencia de castigos), muestra que las cárceles concesionadas poseen un promedio mayor número de castigos $(0,5)$ que las tradicionales $(0,4)$. Así, es posible constatar que dentro del sistema concesionado el sistema de castigos persiste con mayor fuerza que en los centros penitenciarios tradicionales $(\mathrm{p}=0,0$; $\mathrm{t}=-2,5)$ al $95 \%$ de confianza.

Finalmente, en relación a la violencia en los allanamientos, es posible que el promedio es igual en las cárceles concesionadas y en las tradicionales $(0,5)$ de la muestra. Considerando que la variable está medida con el valor 0 para la ausencia de violencia y valor 1 para la presencia de ésta, es posible constatar que existen diferencias estadísticamente significativas.

\subsection{Acceso a programas}

En cuanto al acceso a programas de reinserción en la cárcel, se presentan datos de la Primera Encuesta de Calidad de Vida Penitenciaria. Esta variable fue un indicador compuesto que iba de 0-6, donde 0 representa participación en ningún programa y 6 una participación en 6 programas diferentes, incluyendo capacitación laboral, trabajo remunerado, actividades deportivas, asistencia a la escuela penal, taller psico-social y taller artístico-cultural. El resultado muestra que el promedio de acceso en las cárceles concesionadas fue de 1,5 mientras que, en el caso de las cárceles tradicionales, el promedio fue de 1,8. Esta diferencia fue estadísticamente significativa y representa evidencia preliminar de que las cárceles concesionadas presentarían un menor de acceso a programas de reinserción social en comparación con las tradicionales $(\mathrm{p}=0,0 ; \mathrm{t}=0,2)$.

Ahora bien, para conocer en qué aspectos específicos de los programas ambos sistemas diferían, se llevaron a cabo una serie de análisis de pruebas t para muestras independientes (ver tabla 3, más abajo). En ellos, aparecen diferencias estadísticamente significativas (al 95\% de confianza) en favor de las cárceles tradicionales en tres de los seis programas 
SANHUEZA, Guillermo; PÉREZ, Francisca "Cárceles concesionadas en Chile: evidencia empírica y perspectivas futuras a 10 años de su creación”.

considerados: i) porcentaje de internos que participan de programas de trabajo remunerado ( $31,5 \%$ en tradicionales y $21,5 \%$ en concesionadas; $\mathrm{p}=0.0)$, ii) porcentaje de internos que participan de actividades deportivas $(45,5 \%$ en tradicionales y un $36,7 \%$ entre las concesionadas; $\mathrm{p}=0.0)$, y iii) porcentaje de internos que participan de actividades artísticas o culturales $(20,4 \%$ en cárceles tradicionales y $13,9 \%$ en el caso de las concesionadas; $\mathrm{p}=0.0$ ), todas significativas al $95 \%$ de confianza.

Para el resto de los programas que componían el índice de acceso a programas (capacitación laboral; asistencia a la escuela penal; talleres psicológicos o sociales; taller de reunificación familiar) no se observaron diferencias estadísticamente significativas entre ambos sistemas.

Tabla 3: Indicadores desagregados de participación en programas de reinserción según tipo de cárcel

\begin{tabular}{|c|c|c|c|c|c|}
\hline Ítem & $\%$ Total & $\begin{array}{c}\text { \% Cárcel } \\
\text { Concesionada }\end{array}$ & $\begin{array}{l}\text { \% Cárcel } \\
\text { Tradicional }\end{array}$ & Sig & $\mathbf{N}$ total \\
\hline $\begin{array}{l}\text { Participación en programas de } \\
\text { capacitación laboral }\end{array}$ & $29,2 \%$ & $27,93 \%$ & $29,35 \%$ & & 2.031 \\
\hline Presencia de trabajo remunerado & $30,46 \%$ & $21,52 \%$ & $31,58 \%$ & $* * *$ & 2.009 \\
\hline Asistencia a la escuela penal & $32,47 \%$ & $28,64 \%$ & $32,94 \%$ & & 2.011 \\
\hline $\begin{array}{l}\text { Participación de talleres } \\
\text { psicológicos o sociales }\end{array}$ & $22,98 \%$ & $23,32 \%$ & $22,94 \%$ & & 2.028 \\
\hline $\begin{array}{l}\text { Participación en actividades } \\
\text { deportivas }\end{array}$ & $44,57 \%$ & $36,77 \%$ & $45,53 \%$ & $* *$ & 2.035 \\
\hline $\begin{array}{l}\text { Participación en actividades } \\
\text { artísticas o culturales }\end{array}$ & $19,7 \%$ & $13,96 \%$ & $20,41 \%$ & $* *$ & 2.030 \\
\hline
\end{tabular}

Fuente: Elaboración propia a partir de Encuesta de Percepción de Calidad de Vida en Personas Privadas de Libertad 2013. (*) Significancia al 90\% de confianza (**) Significativo al 95\% de confianza (***) Significativo al $99 \%$ de confianza

Un informe de la DIPRES que busca evaluar los programas de rehabilitación y reinserción social mostraba además que en las Cárceles Concesionadas, la tasa de internos por cada asistente social es menor a la que tendrían las cárceles tradicionales ${ }^{19}$. Estos hallazgos coinciden parcialmente con lo encontrado por Oliveri, en el sentido que no ha sido posible constatar diferencias entre cárceles concesionadas y privadas en el diseño de los programas de reinserción, ni tampoco ha sido posible conocer los motivos por los que no se han cumplido las metas de los concesionados en ese aspecto. Oliveri también evidenciaba una alta rotación de los profesionales y equipos técnicos, lo que dificulta las intervenciones psico-sociales a los internos. Asimismo, reconocía la necesidad de profesionales altamente especializados y un aumento del número de profesionales enfocados en labores de

${ }^{18}$ DIPRES, "Resumen Ejecutivo Evaluación de Programas de Rehabilitación y Reinserción Social", 2012, disponible en: http://www.dipres.gob.cl/595/articles-141207_r_ejecutivo_institucional.pdf [última visita Agosto 2017]. 
Polít. crim. Vol. 12, Nº 24 (Diciembre 2017), Art. 11, pp. 1066-1084.

[http://www.politicacriminal.cl/Vol_12/n_24/Vol12N24A11.pdf]

reinserción, como también aumentar el porcentaje del presupuesto designado para ese quehacer. Así, en las cárceles concesionadas habría una oferta programática que no se estaría adaptando a realidades nacionales y locales, faltando prestaciones y profesionales para generar un adecuado plan de rehabilitación y reinserción.

De todos modos, los porcentajes de internos que han participado de programas del sistema en su totalidad presentan porcentajes relativamente bajos. Aunque de la encuesta no se conocen las razones de la no-participación, una de las hipótesis que explicaría estos bajos guarismos tendría que ver con la poca centralidad que tiene actualmente la labor de reinserción de los centros penitenciarios.

De acuerdo al Instituto Nacional de Derechos Humanos en su Informe de Condiciones Carcelarias 2013, si bien en las cárceles concesionadas la mayoría de los/as internos/as cuenta con un diagnóstico psicosocial (el cual es elaborado al momento de ingresar a cumplir la condena al establecimiento), los/as internos/as declararon en su mayoría que no cuentan con oferta psicosocial permanente, sino solo cuando tienen cumplido el tiempo mínimo para postular a beneficios intrapenitenciarios. En otras palabras, los internos/as se quejaban de recibir escasa atención por parte de psicólogos/as y asistentes sociales.

A partir de los datos recolectados, el acceso a los programas de reinserción constituye, quizás, el aspecto más problemático de las cárceles privadas pues, en ninguno de los seis programas que forman el índice las cárceles privadas presentan porcentajes de participación mejores que sus contrapartes públicas tradicionales; en tres de ellos, inclusive, el sistema penitenciario tradicional muestra mayor participación (trabajo remunerado, actividades deportivas y actividades artístico-culturales). Esto adquiere particular relevancia en tanto las expectativas del sistema penal concesionado en el país tenían una fuerte inclinación hacia que se potencien los programas de reinserción y rehabilitación, posicionándose como una de las principales promesas de estos.

\subsection{Rendición de cuentas y costos}

A partir de la evaluación realizada por Paz Ciudadana el año 2013 es posible considerar que el tema de la rendición de cuentas sería una complicación de este sistema en tanto el estado desconoce la manera en que efectivamente se llevan a cabo los recintos. El mismo informe de Paz Ciudadana del año 2013 tematiza el riesgo de que exista lucro o generación de ganancias con el sistema penal: existe un conflicto de intereses entre la calidad de los servicios entregados y las motivaciones de generar la mayor ganancia a costa de estándares que se degraden. Finalmente, dicha evaluación señala que la privatización de los centros penales dificulta la coordinación de otras agencias públicas, además de dejar en evidencia que se corre el riesgo de dar un giro desde el monopolio del estado hacia el monopolio de un privado.

De acuerdo a Prado, “(...) la alternativa de solución concesionada no ha resuelto el problema hasta la fecha: no ha cumplido con las metas y plazos programados; además de 
SANHUEZA, Guillermo; PÉREZ, Francisca "Cárceles concesionadas en Chile: evidencia empírica y perspectivas futuras a 10 años de su creación”.

tener un costo mayor al de las cárceles tradicionales" ${ }^{20}$ cuestionándose, por ende, la eficiencia y eficacia en la que estaban puestas las expectativas de la colaboración de los privados al quehacer público en el sistema carcelario. Esto, tomando en consideración que se esperaba que las cárceles concesionadas albergaran al 34\% de toda la población privada de libertad ${ }^{3}$ y actualmente solamente albergan al 24,6\%.

\subsection{Reincidencia}

Dentro de los pocos estudios que se tienen noticia sobre la evaluación de las cárceles concesionadas en Chile se encuentra el trabajo realizado por la Fundación Paz Ciudadana el año 2013, donde se compararon los niveles de reincidencia delictual por tipo de cárcel (pública v/s concesionada). Dentro de los principales hallazgos estaba el hecho de que los niveles de reincidencia no presentaban diferencias significativas asociadas al tipo de cárcel que habitaron.

La metodología utilizada en dicho estudio fue cuasi experimental como respuesta a la imposibilidad de contar con dos grupos asignados completamente asignados al azar en el sistema penitenciario chileno. Entre las ventajas de usar cuasi-experimentos se cuenta que proveen una buena alternativa cuando la aleatoriedad no es posible (como es el caso de las cárceles chilenas), siendo recomendados para evaluar resultados a nivel de programas. De este modo, el estudio intentó controlar efectos del recinto y de variables individuales en la reincidencia, ofreciendo una primera aproximación confiable a la pregunta acerca de los niveles de reincidencia en cárceles públicas versus concesionadas.

Otro estudio de reincidencia realizado por Gendarmería de Chile en 2013 mostraba que existían diferencias estadísticamente significativas en la tasa de reincidencia entre ambas modalidades de cárceles, posicionando a las concesionadas casi 3 puntos porcentuales por encima de su contraparte tradicional. La tasa de un $41,1 \%$ de reincidencia para las concesionadas se contrapone al 38,2\% en el caso de las cárceles tradicionales (chi: 0,003), y dichos resultados se dan incluso tras comprobar que variables tales como el compromiso delictual, el tiempo de condena y la conducta de los internos se comporta de manera homogénea para ambos tipos de administración. Un elemento a tener en consideración es que este estudio realizó un periodo de seguimiento de dos años, versus los tres años que, usualmente, son los empleados en este tipo de análisis.

Dicho de otra forma, aun cuando debido a factores como la asignación no-aleatoria de los reclusos ninguno de los dos estudios citados logró aislar totalmente los posibles efectos de variables individuales en los niveles de reincidencia futuras y/o el "efecto cárcel" (multinivel) sobre las trayectorias individuales, la evidencia empírica disponible a partir de ambos trabajos respecto a la efectividad de las cárceles concesionadas en cuanto a disminuir la reincidencia sugiere que no habrían diferencias entre ambos sistemas, lo cual supone un revés a la promesa inicial del sistema concesionado de brindar más reinserción.

\footnotetext{
${ }^{20}$ PRADO, "El sistema penitenciario chileno", cit. nota $n^{\circ} 2$.
} 
Polít. crim. Vol. 12, № 24 (Diciembre 2017), Art. 11, pp. 1066-1084.

[http://www.politicacriminal.cl/Vol_12/n_24/Vol12N24A11.pdf]

En este sentido, futuros estudios podrían incorporar análisis multinivel y/o estudios longitudinales, con el fin de profundizar los análisis y robustecer las conclusiones respecto a la influencia de los recintos, incorporando a su vez análisis adicionales respecto a otras variables -como el vecindario al cual llegan-que impactan en los procesos de reinserción.

\section{Conclusiones y discusión}

El propósito central de este artículo era ofrecer un análisis del sistema de concesiones penitenciarias a 10 años de su creación, fundamentalmente en base a datos secundarios. Hicimos este artículo pensando en la necesidad de un debate sincero en torno al rol que se espera de los privados en cuanto a la política penitenciaria y la reinserción social, dilucidando y explicitando la filosofía detrás de la existencia de este sistema y las expectativas frente a este para los próximos años. En otras palabras, si el país ha de tener cárceles concesionadas, es importante clarificar y explicitar qué debería esperarse de ellas.

La incorporación de actores privados en la construcción y administración de cárceles ha traído mejoras visibles en términos de infraestructura penitenciaria. Así, dentro de las fortalezas del sistema de concesiones destaca la buena percepción de los internos respecto a aspectos de la infraestructura tales como el espacio y privacidad de las celdas, el estado de los baños, la posibilidad de ducharse diariamente y el poder dormir en un lugar limpio.

Por otra parte, y de acuerdo a la evidencia empírica recolectada, algunos nudos críticos del sistema concesionado tienen que ver con el porcentaje de internos que han reportado maltrato de los funcionarios penitenciarios; con el acceso y calidad a servicios de salud; y con el acceso y calidad de la oferta de intervención psicosocial para los internos/as, todo lo cual parece está mejor posicionado en el sistema tradicional que en el concesionado y que amerita una revisión en el marco de las expectativas que se tenían en torno a las cárceles concesionadas en sus inicios.

En lo relativo al acceso a programas de reinserción, es posible constatar que en ninguno de los indicadores analizados se cumple de manera significativa una de las principales promesas de este sistema: mejorar las intervenciones encaminadas a la reinserción social. Así, los beneficios de flexibilidad y mejoras en la gestión que el sistema concesionado iba a enfocar en los programas de reinserción, no han sido corroborados desde el presente estudio.

Los estudios empíricos en cuanto a los niveles de reincidencia resultados poco concluyentes, tanto en Chile como en la evidencia internacional. Por lo general, el indicador utilizado para evaluar el desempeño de las intervenciones de reinserción y rehabilitación es la reincidencia delictual, la cual la cual tiene una gran limitación metodológica en estos casos debido a que la asignación de individuos a los centros penitenciarios no es aleatoria.

La realización de evaluaciones comparativas y debates profundos en este tema permitirán el debido encauzamiento de los esfuerzos y las modificaciones a realizar para lograr las metas sociales y políticas en torno al quehacer penitenciario. Es necesario comprender esto en tanto “(...) una política no solo implica el uso de la autoridad del Estado, sino que es el 
SANHUEZA, Guillermo; PÉREZ, Francisca "Cárceles concesionadas en Chile: evidencia empírica y perspectivas futuras a 10 años de su creación”.

reflejo del choque de intereses en conflicto y de consecuencias distributivas (...); es decir, las decisiones políticas y éticas, en conjunto con las técnicas, modelan cada política" ${ }^{21}$. Pues para lograr cambios reales en materia de política pública penitenciaria, resulta necesario que exista debate y los diversos actores involucrados aporten a profundizar el intercambio de ideas y la solución más coherente con la sociedad.

En este sentido, pensamos que es importante examinar los incentivos que tienen las cárceles para rehabilitar a los internos. Aunque este es un llamado general al sistema penitenciario, este se da con mayor énfasis para el caso de las concesionadas en tanto cuentan con una infraestructura y tecnología que lo permite y también porque son un espacio de innovación que posibilita la concreción de mejoras en aspectos en que el sistema tradicional no está respondiendo de la manera más adecuada.

Quizás, como no queda tan claro de la evidencia empírica revisada si los internos de cárceles concesionadas están reincidiendo menos que aquellos que cumplen condenas en cárceles públicas, sea el momento de pensar en nuevos indicadores de performance que sean proxies de reinserción social, tal como lo propone Liebling con su concepto de "desempeño moral de las cárceles" o como señalaba Dammert y Díaz ${ }^{22}$ en el sentido de "invertir en presos y no en cárceles".

Es necesario que la reinserción sea un tema central dentro del quehacer de las cárceles concesionadas y los esfuerzos tanto desde Gendarmería como desde los grupos de concesiones vayan en esa línea, de manera coordinada y efectiva. En esta línea, sostenemos que las cárceles concesionadas necesitan establecer y/o mejorar los incentivos para reclutar y mantener buenos profesionales dentro de sus equipos técnicos. Esto va enmarcado en que las intervenciones psicosociales requieren de la permanencia de los profesionales de calidad y dada la evidencia proporcionada por Paz Ciudadana y Oliveri de que comparativamente los recintos concesionados presentan mayor rotación de profesionales que los recintos tradicionales.

Además, a partir de una serie de entrevistas informales con personal penitenciario que trabaja en cárceles concesionadas, se recalca la importancia de reforzar la coordinación interna de Gendarmería de Chile con las empresas concesionarias; solo con una sincronía en el quehacer de ambos actores es posible alcanzar un funcionamiento adecuado de los centros penitenciarios y lograr el cumplimiento adecuado de las metas existentes en torno a la realidad carcelaria y la reinserción, combinando así lo mejor de la gestión de los públicos y los privados.

También pensamos que sería provechosa la existencia de una mayor proactividad del sistema concesionado para coordinarse con actores fuera del sistema penitenciario, incluyendo municipios y empresas privadas. Esta coordinación de los actores sería provechosa para el cumplimiento del propósito de reinserción social, gestión carcelaria y

${ }^{21}$ CEJUDO, Guillermo y MICHEL, Cynthia, "Coherencia y políticas públicas: instrumentos y poblaciones objetivos", Gestión y política pública, Vol. 25, № 1 (2016), pp. 3-31, p-21.

${ }^{22}$ DAMMERT, Lucía y DÍAZ, Javiera, "El Costo de Encarcelar"en Programa de Seguridad y Ciudadanía FLACSO, (2005). Santiago, Chile. 
Polít. crim. Vol. 12, Nº 24 (Diciembre 2017), Art. 11, pp. 1066-1084.

[http://www.politicacriminal.cl/Vol_12/n_24/Vol12N24A11.pdf]

calidad de vida penitenciaria entre otros aspectos, enmarcando así una política social carcelaria con actores múltiples y diversos, con miras a la reinserción social efectiva.

Este estudio analizó el funcionamiento del sistema concesionado en base a evidencia empírica de fuentes secundarias. Estos hallazgos, no obstante, deben ser entendidos en el contexto de algunas limitaciones: en primer lugar, la mayor parte de la evidencia empírica analizada corresponde a estudios transversales, lo cual limita la posibilidad de examinar tendencias. En segundo lugar, la mayor parte de la (escasa) evidencia empírica es cuantitativa y, en este sentido, nuevos estudios de tipo más cualitativos o mixtos pudieran aportar a responder algunas preguntas relativas al cómo o porqués de algunas asociaciones que resultaron significativas estadísticamente. En tercer lugar, es posible que el efecto positivo de programas o intervenciones específicas que pudieran estar ocurriendo al interior de algunas cárceles concesionadas permanezcan aún invisibles en medio de datos agregados a nivel de toda una cárcel.

Dadas estas limitaciones resulta aventurado para nosotros emitir una opinión acerca de si se debe o no terminar el sistema privado de cárceles en Chile. Si la concesión de cárceles ha de seguir operando, nuestros hallazgos sugieren que este sistema tiene aún gran parte de su inicial promesa sin cumplir y si este modelo ha de mantenerse, deberían hacerse importantes mejoras en diversos aspectos de su funcionamiento. Así, por ejemplo, se podrían perfeccionar los incentivos para que el privado invierta en presos y no en cárceles ${ }^{18}$ y se logren, de este modo, avances más efectivos en materia de reinserción social y disminución de la reincidencia.

\section{Agradecimientos}

Este artículo fue posible gracias al financiamiento de Conicyt, a través de Fondecyt Iniciación \#11150840. 
SANHUEZA, Guillermo; PÉREZ, Francisca "Cárceles concesionadas en Chile: evidencia empírica y perspectivas futuras a 10 años de su creación”.

\section{BIBLIOGRAFÍA}

ARELlANO, Jaime, Reforma Penitenciaria: El caso del programa de concesiones en infraestructura penitenciaria en Chile, documento emitido en el Seminario Interamericano "Justicia y Gobernabilidad Democrática”, Santiago, Chile, 2003.

ARRIAGADA, Isabel, "De cárceles y concesiones: Privatización carcelaria y penalidad neoliberal", Revista de Derecho de Valdivia, Vol. XXV (2012).

BURÓN, Javier Nistal, "El futuro de la cárcel y la cárcel del futuro", Quadernos de criminología: revista de criminología y ciencias forenses $\mathrm{N}^{\circ} 20$ (2013), pp. 34-41.

CEJUDO, Guillermo y MICHEL, Cynthua, "Coherencia y políticas públicas: instrumentos y poblaciones objetivos”, Gestión y política pública Vol. 25, N¹(2016), pp. 3-31.

DAMMERT, Lucía y DÍAZ, Javiera, "El Costo de Encarcelar"en Programa de Seguridad y Ciudadanía - FLACSO, 2005, Santiago, Chile.

DIPRES, "Resumen Ejecutivo Evaluación de Programas de Rehabilitación y Reinserción Social", 2012, disponible en: http://www.dipres.gob.cl/595/articles141207 r ejecutivo institucional.pdf [última visita Agosto 2017].

DUNCAN, Jeanine, "Report on corrections and the private sector", en: Comission on criminal and juvenile justice, 1986, disponible en: https://www.ncjrs.gov/pdffiles1/Photocopy/102380NCJRS.pdf [última visita Agosto 2017].

ESPINOZA, Olga; MARTÍNEZ, Fernando; SANHUEZA, Guillermo, "El sistema penitenciario y su impacto en las personas privadas de libertad", en: VV.AA., Informe sobre los Derechos Humanos en Chile (2014).

FUNDACION PAZ CIUDADANA Y BID, "Evaluación del sistema concesionado versus el sistema tradicional en la reducción de la reincidencia delictual”, 2013, disponible en: $\quad$ http://www.pazciudadana.cl/wp-content/uploads/2013/07/2013-0702_Evaluaci\%C3\%83\%C2\%B3n-del-sistema-concesionado-versus-el-sistematradicional-en-la-reducci\%C3\%83\%C2\%B3n-de-la-reincidencia-delictual.pdf [última visita Agosto 2017].

GENDARMERÍA DE CHILE, "Boletín Estadístico Chile: Situación carcelaria en Cifras", $\mathrm{N}^{\circ} \quad 01, \quad$ octubre de 2016, disponible en http://static.emol.cl/emol50/documentos/archivos/2016/11/02/20161102115114.pdf [última visita Agosto 2017].

LIEBLING, Alison; CREWE, Ben, "Prisons beyond the New Penology: The Shifting Moral Foundations of Prison Management" en: SIMON, Jonathan; SPARKS, Richard (Eds.), The Sage Handbook of Punishment and Society, Londres: Sage Publications Ltd., 2012, pp. 283-307.

LIEBLING, Alison; LUDLOW, Amy, "Privatising public prisons: Penality, law and practice", Australian \& New Zealand Journal of Criminology (2016).

MARTÍNEZ, Fernando; ESPINOZA, Olga, "Cárceles concesionadas en Chile: ¿El camino a la privatización?", Revista electrónica Debates Penitenciarios, Área de Estudios Penitenciarios CESC, Vol. 9 (2009).

MORALES, Ana María, et al., "La Reincidencia en el Sistema Penitenciario Chileno", Santiago: Fundación Paz Ciudadana, 2013.

OLIVERI, Katherine, "Programas de rehabilitación y reinserción de los sistemas de cárceles", Sociedad Chilena de Políticas Públicas (2011) Santiago, Chile, disponible 
Polít. crim. Vol. 12, Nº 24 (Diciembre 2017), Art. 11, pp. 1066-1084.

[http://www.politicacriminal.cl/Vol_12/n_24/Vol12N24A11.pdf]

en:

http://www.sociedadpoliticaspublicas.cl/archivos/BLOQUET/Seguridad_Ciudadana y_Justicia/Programas\%20de\%20rehabilitacion\%20y\%20reincersion\%20en\%20carcel es\%20concesionadas.pdf [última visita Agosto 2017].

PAVIĆ, Ivica, "Perspectives of Prison Privatization as a Solution to the Prison System Crisis in Croatia", European Journal of multidisciplinary studies, Vol. 3, $\mathrm{N}^{\circ} 1$ (2016).

PRADO, Francisco, "El sistema penitenciario chileno, una aproximación crítica", Revista electrónica Agenda Pública, Vol. V (2006).

PRATT, Travis; MAAHS, Jeff, "Are Private Prisons More Cost-Effective Than Public Prisons? A Meta-Analysis of Evaluation Research Studies", Crime \& Delinquency Vol. 45, N³ (1999), pp. 358-371.

RIBERA, Teodoro, "Realidad actual y desafíos del sistema penitenciario chileno", en: VV.AA., Seminario Cárceles concesionadas revitalizando el modelo organizado por el Instituto Libertad y Desarrollo (2012). Santiago, Chile.

SANHUEZA, Guillermo, "Reinserción Social: el desempeño moral de las cárceles en Chile", Nova Criminis $\mathrm{N}^{\circ} 9$ (2015), pp. 181-209.

UNIVERSIDAD DIEGO PORTALES, Facultad de Derecho, Informe anual sobre derechos humanos en Chile 2008. 